\title{
ON INDIVIDUAL SENSIBILITY TO PAIN. ${ }^{1}$
}

\author{
BY DR. HAROLD GRIFFING.
}

The relative sensibility of individuals to pain is a problem of practical as well as theoretical interest. A more definite knowledge of the subject might be utilized not only in medicine but also in education. The data obtained are, however, quite limited; and most of the observations published have been made by anthropologists with but little consideration of the sources of error involved. The experiments here reported were undertaken with a view to throwing light on these sources of error rather than for statistical purposes.

The tests were made with Professor Cattell's pressure algometer and with the induction coil. The algometer registered the pressure exerted up to I 5 kilo. It was applied by me on the palm of the hand and the forehead in most of the experiments; but in some, the latter ones, the observer applied the pressure himself, and the fleshy part of the thumb was used instead of the palm of the hand. The pressure was increased slowly at a rate as constant as practicable until the sensation became uncomfortable.

In the electrical tests the two forefingers of each hand were placed in separate cups of water and the alternating current was sent through the body from hand to hand. Four gravity cells were used with an induction coil. The distance of the primary coil from the secondary served as a rough indicator of the electromotive force of the current, which may be taken to represent the intensity of the stimulus.

The purpose of the first group of experiments was principally to find whether the sensibility of a sense organ might not be determined, in whole or in part, by the degree of protection given by other tissues. If decreased sensibility to pain may be due to the thickness of the skin, the determination of the dermal threshold for pain tells us nothing of a person's general sensibility to

2 From the Psychological Laboratory of Columbia University. 
pain. In order to investigate this source of error, before making the test with the algometer, I recorded my judgment of the probable result from the appearance of the hand. This was done on fifty-three students, four tests being made on each person, two on each hand. The men were marked $a, b, c$ or $d$, according to their estimated sensibility. I give below the resulting average values of the pain threshold in kilograms, with the maxima and minima. The results are given in two groups, I. and II., since in group II., the observer applied the stimulus to the thumb, whereas in I., I applied it myself to the palm. Some of the tests in II. were kindly made for me by advanced students in the Columbia Psychological Laboratory in connection with other independent tests.

TABLE I.

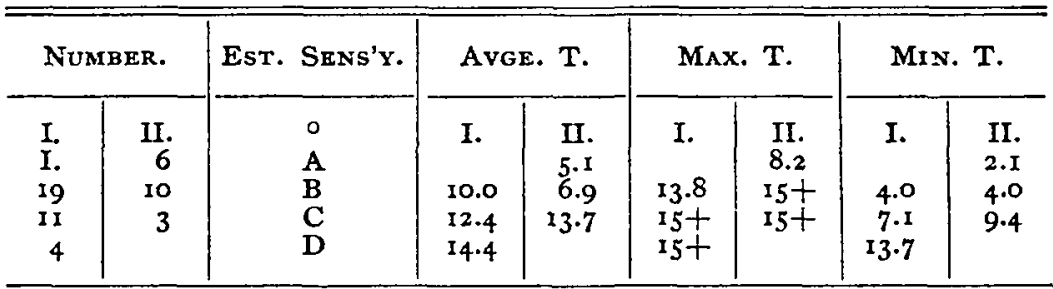

First vertical column gives number of observers; the second gives the estimated sensibility, the others the average and maximum and minimum values of the pain threshold $(\mathrm{T})$.

From the results above given it is evident that, as might be expected, the thickness of the skin and subcutaneous tissues is an important element in determining the threshold for dermal pain. It is not, however, the only element involved. Some observers were much more sensitive and others less sensitive than one might expect from the appearance of the hand.

If the protection given by the tissues varies with individuals, then the relative sensibility of different parts of the body will presumably also be subject to individual variations. This I found to be true. Those having a high pain threshold for the hand were not always correspondingly sensitive to pressure applied to the forehead and top of the head.

Nevertheless, as will be seen by the following table, those who were sensitive on the hand were on the average more sen- 
sitive on the head. I give now the average values, with maximum and minima, of the pain threshold for the head, for the different sets rated according to the value of their hand threshold. The first half of the table is for the men classed group I. in the previous table, and the second is for those classed in group II. The top of the head was used with group II., the forehead for group I.

TABLE II.

\begin{tabular}{|c|c|c|c|c|}
\hline Number. & T. FOR HAND. & T. FOR HEAD. & Max. & Mxs. \\
\hline $\begin{array}{r}12 \\
15 \\
12 \\
3 \\
6 \\
14\end{array}$ & $\begin{array}{c}\text { Over I3 } \\
\text { 10 to I3 } \\
\text { Under Io } \\
\text { Over Io } \\
6 \text { to Io } \\
\text { Under } 6\end{array}$ & $\begin{array}{l}6.5 \\
5.0 \\
3.5 \\
7.0 \\
2.3 \\
1.9\end{array}$ & $\begin{array}{r}13.4 \\
5.7 \\
4.6 \\
11.5 \\
3.5 \\
5.1\end{array}$ & $\begin{array}{l}3.2 \\
3.4 \\
1.5 \\
4.8 \\
1.1 \\
1.0\end{array}$ \\
\hline
\end{tabular}

The first vertical column gives number of observers.

The second vertical column gives threshhold values for three groups in order of sensibility.

The third, fourth, and fifth columns give average threshhold and maximum and minimum values for observers mentioned in second column.

It has generally been assumed that the sensibility, to one form of painful stimulation may serve as an index to pain sensibility in general. But there is no evidence for such an assumption. In order to obtain data on the subject, I tested 27 observers with the induction coil as well as with the pressure algometer. In this way I found that the sensibility to electrical stimulation to be quite independent of pressure sensibility. Three observers, among the most sensitive to pressure on the hand and head declare, that they felt no discomfort at all when the maximum strength of current was given. One of these did not 'feel' the current when six cells were used instead of four, although the muscles of his fingers contracted from the stimulation of the current. On the other hand one student to whom a pressure of $15+$ kilo. on the hand and II kilo. on the head, gave no discomfort, considered the electrical effect unpleasant when the current was of the average strength.

The above experiments do not, however, prove that the pain sensibility of the nervous system to different forms of stimu- 
lation is not the same. We can only conclude that the pain sensibility to sensory stimulation varies with the conditions of stimulation. Not knowing the path of the current in the body, we have no right to assume any special physiological or psychological basis for the data obtained from introspection.

The probable fact that in the pressure experiments, the variations cannot all be explained by the thickness of the skin and similar conditions, goes to show that there is such a thing as general sensibility to pain; and the general correspondence of the results for different places of stimulation may be interpreted in the same way. But even these results may be due to peripheral causes, and not to any property of the central nervous system, or of the consciousness by which it is accompanied. 